\title{
Annealing effects on magneto-optical Kerr effect and magnetic anisotropy in ultrathin Co films on $\operatorname{Pt}\left(\begin{array}{lll}1 & 1 & 1\end{array}\right)$
}

\author{
M.-T. Lin ${ }^{\mathrm{a}, *}$, H.Y. Her ${ }^{\mathrm{b}}$, Y.E. Wu ${ }^{\mathrm{b}}$, C.S. Shern ${ }^{\mathrm{b}}$, J.W. Ho ${ }^{\mathrm{a}}$, C.C. Kuo ${ }^{\mathrm{a}}$, H.L. Huang ${ }^{\mathrm{a}}$ \\ ${ }^{a}$ Department of Physics, National Taiwan University, Taipei 106, Taiwan \\ bepartment of Physics, National Taiwan Normal University, Taipei 117, Taiwan
}

\begin{abstract}
Large change in magneto-optical Kerr response and increase in perpendicular anisotropy were observed in ultrathin Co films on $\mathrm{Pt}\left(\begin{array}{lll}1 & 1 & 1\end{array}\right)$ upon thermal annealing at elevated temperatures. Monitoring the film structure by using low-energy electron diffraction and Auger electron spectroscopy, these findings were found to be correlated to the $\mathrm{Co}-\mathrm{Pt}$ alloy formation at interface. (C) 2000 Elsevier Science B.V. All rights reserved.
\end{abstract}

Keywords: Magneto-optic Kerr effect; Perpendicular magnetic anisotropy; Ultrathin film; Thermal annealing

Co-Pt-based alloy thin films and multilayers have attracted considerable interest due to their promising application in technology of magneto-optical recording media $[1,2]$. As compared to Co-Pt multilayer and alloy systems, there are, up to now, only few studies of $\mathrm{Co} / \mathrm{Pt}$ films in ultrathin scale probably because of their limitation of a direct application. Nevertheless, studying the ultrathin film system could provide the basic and detailed information on the epitaxial process, growth modes, and interface or surface diffusion. In particular, the behavior of the surface or interface alloying may be completely different from those in the bulk. The information obtained in the ultrathin limit study may thus be of much use for further fabricating the Co-Pt multilayer and alloy thin film systems. In this contribution, we report the alloy formation at interface upon thermal annealing for $\mathrm{Co} / \mathrm{Pt}\left(\begin{array}{lll}1 & 1 & 1\end{array}\right)$ ultrathin films, and focus on their enormous influence on the perpendicular magnetic anisotropy and magneto-optical Kerr effect.

The experiments were carried out in an UHV-chamber with base pressure in the range $5 \times 10^{-10}$ mbar. The pressure during Co deposition was kept at about $1 \times 10^{-9}$ mbar at an evaporation rate of about $0.1 \mathrm{ML} / \mathrm{min}$. All

\footnotetext{
* Corresponding author. Fax: + 886-2-23639984.
}

E-mail address: mtlin@phys.ntu.edu.tw (M.-T. Lin)
Co films investigated were grown at $325 \mathrm{~K}$ and then annealed at different temperatures up to $850 \mathrm{~K}$. The crystalline structure and composition of the film surface were checked by low-energy electron diffraction (LEED) and Auger electron spectroscopy (AES), respectively. Magnetic properties were monitored by magneto-optical Kerr effect (MOKE) using a p-polarized $\mathrm{He}-\mathrm{Ne}$ laser $(632.8 \mathrm{~nm})$ as the light source.

Fig. 1 shows the results of measurements of both polar Kerr signal and $\mathrm{Co}_{775 \mathrm{eV}} / \mathrm{Pt}_{237 \mathrm{eV}}$ Auger signal ratio taken at $325 \mathrm{~K}$ of the $1.0 \mathrm{ML}$ film annealed at $600 \mathrm{~K}$ for different annealing times. AES is used here to monitor the depth of diffusion of adsorbate as well as the surface composition during thermal annealing. The $\mathrm{Co}_{775 \mathrm{eV}} /$ $\mathrm{Pt}_{237 \text { ev }}$ Auger signal ratio shown in Fig. 1 drops drastically at the beginning and reaches a minimal value (not zero) after an annealing time above $15 \mathrm{~min}$. As the Auger signal ratio decreases rapidly with increasing time, the polar Kerr remanence signal increases drastically. Corresponding to the stabilization of Auger signal ratio, the polar Kerr remanence signal also reaches a saturated valve about $260 \%$ of that before annealing. The stabilized Auger signal ratio indicates a constant chemical composition and the formation of a metastable phase at interface, as identified more clearly as interface $\mathrm{Co}-\mathrm{Pt}$ alloy later.

Not only the magneto-optical Kerr response but also the perpendicular magnetic anisotropy is enhanced after 


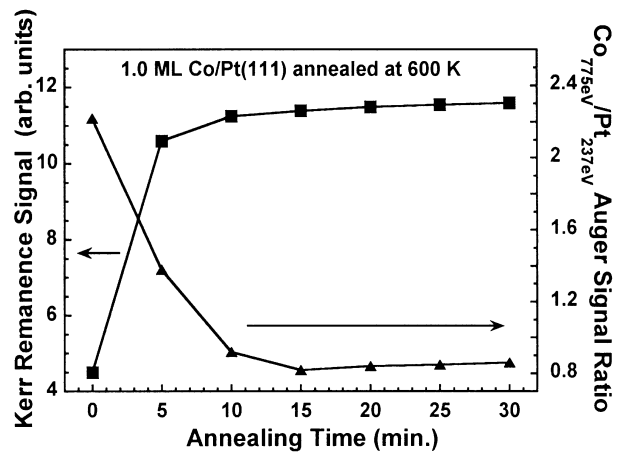

Fig. 1. The polar Kerr remanence signal (square) and the $\mathrm{Co}_{775 \mathrm{eV}} / \mathrm{Pt}_{237 \mathrm{eV}}$ Auger signal ratio (triangles) of the $1.0 \mathrm{ML}$

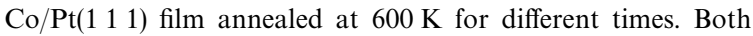
MOKE and AES data were taken at $325 \mathrm{~K}$.

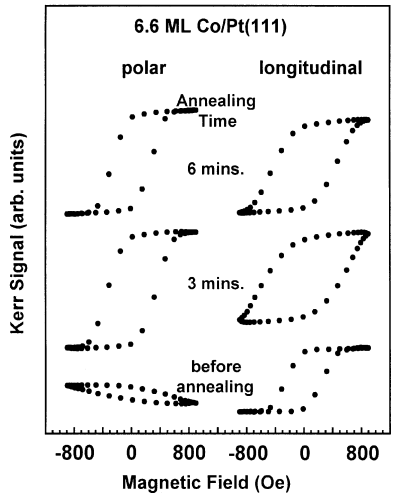

Fig. 2. Compilation of both polar and longitudinal Kerr hysteresis loops for the 6.6 ML Co/Pt(1 111$)$ annealed at $850 \mathrm{~K}$ for different times. The measurement temperature is $325 \mathrm{~K}$.

thermal annealing. Fig. 2 shows the Kerr hysteresis loops for the 6.6 ML annealed at $850 \mathrm{~K}$ for different times. The 6.6 ML as-grown film has an in-plane magnetization with only tiny perpendicular Kerr signal. After 3 min of annealing at $850 \mathrm{~K}$ of the film, the perpendicular Kerr signal increases drastically, while the in-plane hysteresis loop becomes more round as compared to that before annealing, indicating clearly an increase in magnetic perpendicular anisotropy. After 6 min of annealing, both the perpendicular and in-plane Kerr signals become smaller. Since the diffusion mobility at this elevated temperature seems already too high to stabilize a Co-Pt alloy state at the interface, the Co atoms diffuse deeply into the substrate further. This indicates that the annealing temperature is a sensitive parameter for the interdiffusion process for forming interface alloy.

Fig. 3 combines the results of the Kerr remanence signal measured at $325 \mathrm{~K}$ for the $1.0 \mathrm{ML}$ films annealed

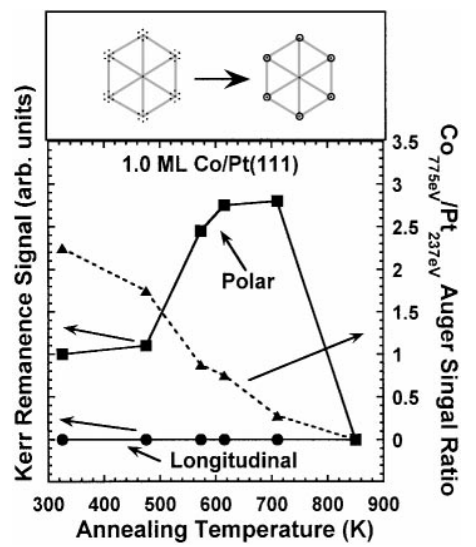

Fig. 3. Combination of magneto-optical Kerr remanence signal (squares and circles for the polar and longitudinal geometry, respectively), the $\mathrm{Co}_{775 \mathrm{eV}} / \mathrm{Pt}_{237 \mathrm{eV}}$ Auger signal ratio (triangles), and the schematic LEED patterns for the $1.0 \mathrm{ML}$ $\mathrm{Co} / \mathrm{Pt}\left(\begin{array}{lll}1 & 1 & 1\end{array}\right)$ annealed at different temperatures for $5 \mathrm{~min}$. The measurement temperature is $325 \mathrm{~K}$.

at different temperatures for $5 \mathrm{~min}$, the $\mathrm{Co}_{775 \mathrm{eV}} / \mathrm{Pt}_{237 \mathrm{eV}}$ Auger signal ratio, and the schematic LEED pattern. An onset of enhancement of the polar Kerr signal is observed at about the annealing temperature of $475 \mathrm{~K}$. The polar Kerr signal is enhanced drastically when the film is annealed above $500 \mathrm{~K}$, and at about $700 \mathrm{~K}$ annealing temperature it reaches a maximal value about $300 \%$ of that before annealing. Accompanying the large enhancement of Kerr signal, a rapid drop of the $\mathrm{Co} / \mathrm{Pt}$ Auger signal ratio is observed, indicating a strong $\mathrm{Co}-\mathrm{Pt}$ interdiffusion at the interface. The LEED patterns in the upper-panel of Fig. 3 reveal two different characteristic structures. The first one for the films with the annealing temperature range up to about $550 \mathrm{~K}$ reveals an incommensurate mode, including a $\mathrm{p}(1 \mathrm{X} 1)$ structure based on symmetry fitted to the $\operatorname{Pt}\left(\begin{array}{lll}1 & 1 & 1\end{array}\right)$ substrate and a sixfold superstructure around each integer spot. This is attributed to an incoherent epitaxy of the Co film on the $\operatorname{Pt}\left(\begin{array}{lll}1 & 1 & 1\end{array}\right)$ surface [3]. For annealing temperatures above $550 \mathrm{~K}$, the LEED patterns become fuzzy and have a $\mathrm{p}(1 \mathrm{X} 1)$ structure with similar value of the lattice constant as the $\operatorname{Pt}\left(\begin{array}{lll}1 & 1 & 1\end{array}\right)$ substrate. Together with the finding of the rapid drops of the $\mathrm{Co} / \mathrm{Pt}$ Auger signal ratio, this structural evolution observed in LEED patterns provides the strong evidence of the Co-Pt alloy formation, which can be stabilized even for a long annealing time below a certain annealing temperature, as mentioned above.

On increasing the annealing temperature further up to $850 \mathrm{~K}$, both the polar and longitudinal Kerr remanence signals vanish completely, indicating disappearance of the ferromagnetic ordering or magnetization. (Note that the longitudinal Kerr remanence signal is zero for all 
annealing temperatures for the $1.0 \mathrm{ML}$ film.) The annealing temperature $850 \mathrm{~K}$ is obviously too high for forming a metastable alloy phase, and the Co atoms diffuse deeply and completely into the $\operatorname{Pt}\left(\begin{array}{lll}1 & 1 & 1\end{array}\right)$ substrate, losing the ferromagnetic ordering.

The correlation of the alloy formation at the interface and the enhancement of the magneto-optical Kerr effect and increase in magnetic perpendicular anisotropy may be mainly traced back to the transfer of the strong spin-orbit coupling of the Pt into the magnetic Co orbital due to the hybridized electronic structure, as suggested in the theoretical study for the Co-Pt ultrathin multilayer system [4]. The contribution of the enhanced Co magnetic moment is, however, only of minor importance. The $300 \%$ maximal value of the enhanced magneto-optical Kerr signal after thermal annealing is an amazing factor as compared to the previous study for the $\mathrm{Co} / \mathrm{Pt}$ multilayer and $\mathrm{Co}-\mathrm{Pt}$ alloy systems [5], in which an overall $50 \%$ enhancement of magneto-optical response is found. The significant enhancement of the magneto-optical Kerr signal in our case may be due to a special hybridization state between the $\mathrm{Co}$ and $\mathrm{Pt}$ through the thermal annealing, which is different from the expected structure in the $\mathrm{Co}-\mathrm{Pt}$ alloy and multilayer systems as usual. This suggests that thermal annealing could be an effective postdeposition processing to optimize the magneto-optical properties and magnetic anisotropy, and may become technologically relevant.
In conclusion, thermal annealing of $\mathrm{Co} / \mathrm{Pt}\left(\begin{array}{lll}1 & 1 & 1\end{array}\right)$ ultrathin films at appropriate temperatures results in a kind of Co-Pt alloy formation at the interface, which in turn, gives rise to a large enhancement of magneto-optical Kerr response up to about $300 \%$ and increase in perpendicular magnetic anisotropy. The highly enhanced magneto-optical response is believed to be mainly attributed to the transfer of the strong spin-orbit coupling of the $\mathrm{Pt}$ into the Co caused by the hybridized electronic state at the alloying interface.

\section{Acknowledgements}

This work was supported by the National Science Council of Taiwan under Grant Nos. NSC 88-2112-M002-035 and NSC 87-2112-M-003-002.

\section{References}

[1] G.R. Harp, D. Weller, T.A. Rabedeau, R.F.C. Farrow, M.F. Toney, Phys. Rev. Lett. 71 (1993) 2493.

[2] T.C. Huang, R. Sayoy, R.F.C. Farrow, R.F. Marks, Appl. Phys. Lett. 62 (1993) 1353.

[3] J.S. Tsay, C.S. Shern, Surf. Sci. 396 (1998) 313.

[4] G.Y. Guo, H. Ebert, J. Magn. Magn. Mater. 156 (1996) 173.

[5] D. Weller, H. Brandle, G. Gorman, C.-J. Lin, H. Notarys, Appl. Phys. Lett. 61 (1992) 2726. 